\title{
Bile Acids Induced Cell Necropotosis
}

\author{
Li Wanwei ${ }^{1}$, Liu Menggang ${ }^{2}$, Chen Long1, Wu Fang ${ }^{1}$, Song Yao1, Shi Yuan ${ }^{1}$ and Hu Zhangxue ${ }^{1 *}$ \\ ${ }^{1}$ Department of Pediatrics, Daping Hospital, Institute of Surgery Research, Third Military Medical University, Chongqing, P.R.China \\ ${ }^{2}$ Department of Hepatobiliary Surgery, Daping Hospital, Institue of Surgery Research, Third Military Medical University, Chongqing, P.R.China
}

\begin{abstract}
Bile acids were confirmed as important signal molecule in a lot of physiological processes. Recent studies have demonstrated their roles in regulating cell death pathway. New concept of programmed cell death includes autophagy and necroptosis, two independent pathway of programmed cell death beside apoptosis. The molecular pathway of bile acid induced apoptosis and autophagy were reported by recent studies. However, bile acid induced necroptosis was only noticed by some clinical reports, the molecular pathway is waiting to be discovered. This review aims to summarize the pathway of bile acid induced apoptosis and autophagy and analyze the possible pathway of bile acid induced necroptosis.
\end{abstract}

Keywords: Bile acids; Cell death; Apoptosis; Autophagy; Necroptosis

\section{Introduction}

Necroptosis is a relatively new conception in cell death. Cell death is one of the basic cell properties, as important as cell growth, cell proliferation and cell differentiation. Programmed Cell Death (PCD) is a critical procedure for the development and homeostasis of multicellular organisms [1]. Three types of pathway involved in PCD have been identified [2] from basic studies: (1) Apoptotic cell death is defined by an ensemble of morphological features, including cellular shrinkage, plasma membrane blebbing, chromatin condensation, nuclear fragmentation, and the formation of apoptotic bodies [3]; (2) Autophagic cell death is associated with the formation of autophagic vacuoles inside the dying cell, following with autophagic protein induced lysosomal rupture [4]; (3) Necrotic cell death was once recognized as a passive procedure, characterized by cellular swelling, plasma-membrane rupture and the subsequent loss of the intracellular contents. Classical necrosis is passive chemical cell death after cellular damage, that is to say, its progress is inevitable. However, new evidence showed that necrosis could also be initiated and develops in a certain pathway. This programmed cell necrosis procedure was termed "necroptosis" by Degterev Alexei, et al. [5-7].

As a group of signal molecules in the body, bile acids act as important inducer of cell death. Classical role of bile acids was presented in the lipid digestion and absorption process. First evidence of bile acids induced cell death was found from the study of cirrhosis. Further study showed bile acids could activate cell death receptor Fas and TRAIL-R2, and start caspase dependent apoptosis. After autophagy was discovered, studies also discover the effect of bile acids to autophagic pathway. Apoptosis and necrosis are two common forms of cell death that play significant roles in developmental and steady homeostasis. Because of the formation of apoptotic bodies, apoptosis has small effect on systemic inflammation activation. Necrosis is morphologically identified by the swelling of organelles, increasing cell volume, disruption of the plasma membrane, and loss of intracellular contents. Because of cell debris increase, it has significant inflammatory effect and could induce Systemic Inflammatory Response Syndrome (SIRS), which could lead to refractory Acute Respiratory Distress Syndrome (ARDS) and Multiple Organ Failure (MOF) [8]. Since cell necroptosis could also be initiated by death receptors and regulated by intracellular signaling molecules [9], it will be interesting to study the role of bile acid in inducing necroptosis.

With the progress in cell necroptosis study, a lot of studies showed necroptosis plays an important role in severe pathological process, such as inflammatory response syndrome (SIRS) and Multiple Organ Failure (MOF). Bile acid induced pneumonia and Acute Respiratory Distress Syndrome (ARDS) were found in clinical cases with unknown pathological pathway. The connection between bile acids induced necroptosis and SIRS could be the molecular mechanism of these diseases.

\section{Cell Death and Necroptosis}

As a basic biological procedure of the cell metabolism, cell death is not only the end of the cells' lives, but also change the structure and function of the organs. Cell death is regulatable as well as cell growth, cell proliferation and differentiation. The classical pathway of programmed cell death is caspase cascade, with or without involvement of mitochondria [3,10]. In contrast to apoptosis, necrosis has been commonly viewed as an accidental and unregulated event [9]. However, increasing evidences indicated that necrosis can also be executed by regulated mechanisms, which is termed as necroptosis. Necroptosis is an alternative receptor-induced form of PCD that is regulated and caspase-independent, with morphological resemblance. This type of cell death is typically seen by the engagement of certain members of the subset of 'death receptors', the TNF superfamily and particularly of the TNF receptor itself, in case of caspase pathway is blocked [11]. Necroptosis exhibits a unique signaling pathway that requires the involvement of the receptor interaction protein kinase 1 and receptor interaction protein kinase 3 (RIPK1 and RIPK3) and that can be specifically inhibited by necrostatins. Necroptosis has been found to contribute to the regulation of immune system, cancer development as well as cellular responses to multiple stresses [12].

RIPK1 and RIPK3 could form a complex and start the signaling pathways [13]. Phosphorylation of RIPK1 and RIPK3 is critical for

*Corresponding author: Hu Zhangxue, Department of Pediatrics, Daping Hospital, Third Military Medical University, 10 Changjiang Branch Road, Chongqing 400042 , China, Tel: 86-023-68757730; Fax: 86-023-68757731; E-mail: huzx1@163.com

Received January 24, 2014; Accepted February 24, 2014; Published February 26,2014

Citation: Wanwei L, Menggang L, Long C, Fang W, Yao S, et al. (2014) Bile Acids Induced Cell Necropotosis. J Cytol Histol S4: 004. doi:10.4172/2157-7099.S4-004

Copyright: @ 2014 Wanwei L, et al. This is an open-access article distributed under the terms of the Creative Commons Attribution License, which permits unrestricted use, distribution, and reproduction in any medium, provided the original author and source are credited. 
assembly of the necrosome, an amyloid-like complex that initiates transmission of the pro-necrotic signal. Moreover, they are central players in TNF-induced programmed necrosis: the RIPK1 determines whether cell goes survival or death, and RIPK3 determines the type of cell death apoptosis or necroptosis [14].

Recent studies demonstrated that RIPK3 is critical in necroptosis and RIPK3 knockdown leads to a notable inhibition of necroptosis in HT-29 cells [15]. RIPK3 is also a molecular switch between dead receptor signaling-induced apoptosis and necroptosis. Cells with a low RIPK3 expression level are resistant to necroptosis, and transfection of these cells with the RIPK3 gene initiates the necroptosis progress, while the apoptotic pathway is blocked [16]. High level of RIPK3 expression cells will initiates necrosis pathway; low level of RIPK3 expression initiates apoptosis pathway [15]. The pseudokinase Mixed Lineage Kinase domain Like protein (MLKL) is a component of the "necrosome," which triggers Tumor Necrosis Factor (TNF)-induced cell death by necroptosis. It functions as a substrate of RIPK3 to mediate downstream signaling of necroptosis [17]. However, the role of MLKL in this process remains unclear. In addition, RIPK3 can also induce apoptosis and directly promote pro-inflammatory cytokine production [18]. Therefore it will be important to determine whether non-necroptotic RIPK3 signal pathway influences RIPK3-dependent pathologies.

\section{Bile Acids and Cell Death}

Bile acids are important physiological agents for intestinal nutrient absorption and biliary secretions, toxic metabolites. Bile acids also are signaling molecule and metabolic regulator through nuclear receptors and G Protein-Coupled Receptor (GPCR), which could regulate hepatic lipid, glucose, and energy homeostasis. Moreover, toxic bile acids may cause inflammation, apoptosis, and cell death [19]. Studies have shown that the accumulation of bile acids in the hepatocyte can induce cytotoxicity through two mechanisms: apoptosis [20] and necrosis [21]. Bile acids have the potential to induce cell death both through nonspecific detergent effects and through receptor-mediated effects. Enterohepatic circulation of bile acids plays a vital role in nutrient absorption and distribution, and metabolic homeostasis. These physiological processes are regulated by a complex membrane transport system in the liver and intestine, which is regulated by nuclear receptors [19] . Bile acids are considered regulatory molecules: they can act as hormones that interact with nuclear receptors, triggering membrane perturbations which could activate pathways involving membrane-associated proteins [22].

Previous studies showed that, Bile acids induced cell death by activating death receptors Fas and TRAIL-R2 [23]. Although Bile acids can't directly combine with the death receptors, they can release Fas proteins stored in the Golgi apparatus and increasing the TRAIL-R2 transcription, thereby increasing the number of death receptors on cell membrane and oligomerization of receptors, which is described in Figure 1 [23]. Oligomerization of death receptors can activate the formation of Death-Inducing Signal Complex (DISC), which induces the transmission of downstream signaling molecules of PCD.

Interestingly, necroptosis and apoptosis both can be activated by death receptors. However, with the combination of death signal molecule and death receptor, only one way of cell death could be activated, apoptosis or necrosis. Although death receptors could initiate programmed cell death, they do not determine the intracellular pathway. DISC has been found as a key compound in the transforming of intracellular cell death pathway. Each component of DISC, caspase- 8
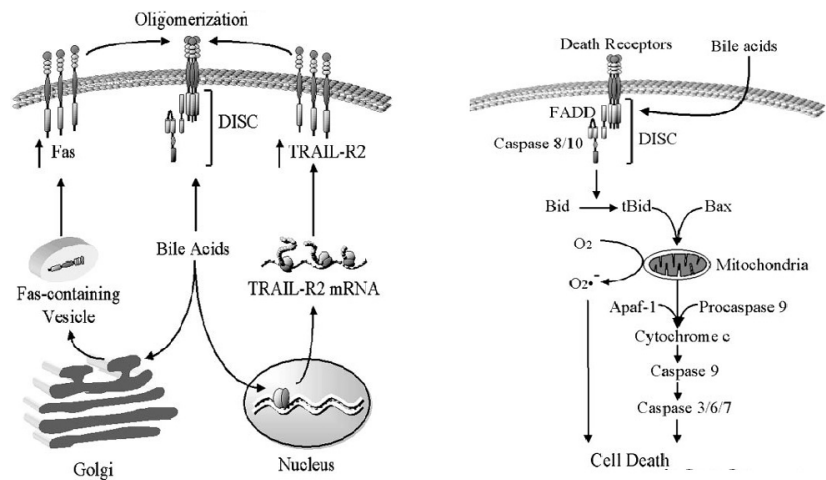

Bile acid induced apoptosis

1) Bile acids promote both Fas and TRAIL-R2/DR5 oligomerization by distinct mechanisms. Bile acids enhance TRAIL-R2/DR5 mRNA transcription and protein expression. In contrast, cellular Fas expression level is not altered by bile acids; however, bile acids stimulate Golgi-associated and microtubuledependent Fas trafficking to the plasma membrane by JNK- and PKCdependent processes, resulting in an increased density of cell surface Fas. The increased cell surface density of these death receptors likely promotes death receptor oligomerization, initiating a DISC formation.

2) Toxic bile acids promote death receptor-mediated cell death signaling Toxic bile acids induce oligomerization of cell surface death receptors. FADD promotes recruitment of procaspase-8/10 to the death-inducing signaling complex (DISC), results in spontaneous activation of caspases via autoproteolytic cleavage, and induce mitochondrial dysfunction including cytochrome $c$ release. Cytosolic cytochrome $c$ then binds to apoptosisactivating factor-1 (Apaf-1), resulting in activation of caspase-9. Caspase-9 can activate effector caspases such as caspases- $3,-6$, and -7 via a caspase cascade, As a result of excess production, oxidative stress may exaggerate cell death, either enhancing apoptosis or inducing necrosis.

Figure 1: Interaction between bile acids and death receptors (left) and bile acid induced cell apoptosis (right).

and Fas Associated Death Domain (FADD) are essential to promoting apoptosis [24]. When bile acid activated death receptor, the downstream of the death pathway is not found yet.

\section{Bile Acids Receptors}

Bile acid receptors are membrane or nuclear receptors which could be activated by bile acids and initiate different pathways. In addition, bile acids can regulate gene expression in the liver and small intestine via activating the nuclear receptors: farnesoid X receptor (FXR; NR1H4), pregnane X receptor (PXR; NR1I2), vitamin D receptor (VDR; NR1I1), G protein coupled receptor TGR5, and some other cell signaling pathways (JNK1/2, AKT and ERK1/2). These nuclear receptors are transcription factors crucially involved in the regulation of bile acids metabolism and detoxification. Among these nuclear receptors, FXR is known to be a major bile acids-responsive ligand-activated transcription factor and a crucial control element for maintaining bile acids homeostasis [25]. It is also well documented that bile acids induce membrane perturbations, which activate different signaling pathways involving PKC and EGFR [26]. To accomplish this regulatory function, bile acids may induce programmed cell death fostering the renewal of the epithelium [27]. Besides Farnesoid X Receptor (FXR) is the crucial nuclear receptor and transcription factors in in the nucleus, it can regulate the metabolism of bile acids, cell death and a series of gene transcription [28]. Moreover, FXR has a high affinity for several major endogenous bile acids: notably cholic acid, deoxycholic acid, chenodeoxycholic acid, and lithocholic acid. Recent evidence suggests that the interaction between bile acids and FXR is involved in the pathophysiology of a wide range of diseases 
of the liver, biliary and gastrointestinal tract, such as cholestasis, liver inflammatory diseases, hepatocellular carcinoma, bowel inflammatory disease and inflammation-associated cancer of the colon and esophagus.

As a transcription factor, FXR binds to DNA either as a monomer or as a heterodimer with retinoid X receptor (RXR, NR2B1) to regulate the expression of various FXR target genes. Furthermore, FXR is highly expressed in the liver, intestine, kidney and adrenals, but with much lower expression in the adipose tissues and heart $[29,30]$. There are four isoforms of FXR identified by either differential use of two promoters or alternative splicing [31,32]. In a number of cells farnesol-induced apoptosis was found to be linked to activation of the apoptosome. We can consider that FXR has very close effect on cell death [33]. Lots of evidence suggests a key role of FXR in both hepatic and intestinal tumorigenesis. Given the crucial role of FXR in maintaining bile acids concentrations within a physiological range, preventing bile acidsinduced cytotoxicity, the loss of FXR would be expected to be associated with a protumorigenic phenotype. FXR activation inducing cell death is varied in the tumor cells [34]. More importantly, FXR could induce cell death in a variety of tumors [28].

\section{Molecular Pathway of Bile Acids Induced Necroptosis}

Expression levels of caspase-8, RIPK3, FLIP, and FADD have been shown as critical regulators of both the apoptotic and necrotic pathways of cell death. When FLIP, FADD or caspase- 8 is absent, RIPK3 activation can initiate necrotic cell death. If RIPK3 is absent, limited FLIP can be permissive for apoptotic activation. It is adequate for caspase-8-dependent apoptosis [35]. Therefore, inhibition of RIPK3 mediated necrosis is possible to treat acute or chronic inflammationrelated diseases such as pancreatitis. Apoptosis and necroptosis show the constrained relationship. Apoptosis-inducing cytokines also cause necrosis in certain cell lines, especially when caspases are inhibited or cannot be activated efficiently. When caspases are inhibited by pharmacological inhibitors or under certain physiological conditions such as viral infections, RIPK1 and RIPK3 form the necrosome to initiate a third pathway known as programmed necrosis or necroptosis [36]. And when the Caspase- 8 or FADD is expressed, RIPK3 induced programmed necrosis is obvious inhibition [37,38]. In contrast, programmed necrosis and necroptosis, which occur only when apoptosis and caspase activity are inhibited, provide an important defense against intracellular pathogens which can suppress classical apoptotic cell death $[39,40]$.

As to bile acids induced cell death, an interesting experiment shows: low concentration of bile acids induce apoptosis, high concentration bile acids induces necroptosis [41]. Because of the type of cell death is not determined by the death receptors, so bile acids if only activate death receptors, it cannot explain this phenomenon has been presented. Bile acids maybe have a direct effect on DISC, another pathway of necroptosis beyond the classical death receptor signaling pathways. After bile acids treatment of The Alveolar Epithelial Cell (AEC), RIPK3 mRNA increased, suggesting bile acids may affect RIPK3 expression. The high concentration of bile acids will not only activate FXR but also significantly increase FXR level in the nucleus [42]. Furthermore, bioinformatics analysis study found that FXR play a vital role in the transcriptional regulation of PIPK3, by the high level of complementarity between the FXR and the regulatory sequences region of RIPK3 gene. In conclusion, bile acids not only can induce cell death that the cell membrane receptor Fas and TRAIL-R2 initiate, but also

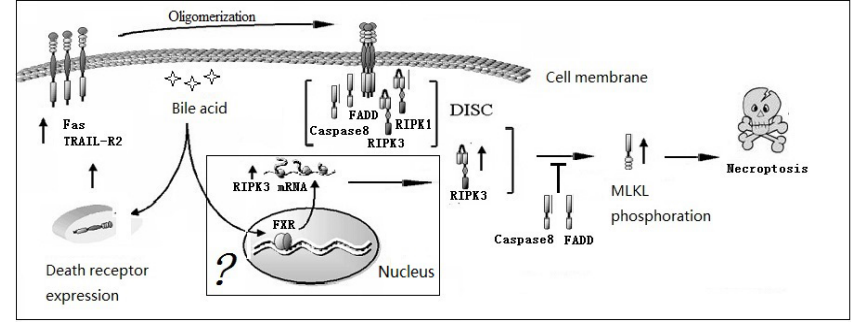

Bile acids induced necroptosis

Bile acids could interact with DISC via 2 independent pathway, 1) Activate death receptors Fas and TRAIL-R2, death receptor oligomerization activate caspase 8 and FADD, this via the way to apoptosis. 2) Activate RIPK3 via FXR, this is the way to necroptosis. The caspase 8 , FADD, RIPK1-RIPK3 compound determine the downstream of cell death. RIPK3 over expression will lead to cell neroptosis directly via MLKL phosphoration

Figure 2: Pathway of bile acids induced necroptosis.

can up-regulate key signaling molecule RIPK3 of necroptosis by FXR, thus bile acids change the way of cell death. The possible pathway of bile acids induced cell necroptosis could be described as Figure 2.

\section{Conclusion}

In the past few years, necroptosis has been proven to play important roles in the regulation of immune system, tissue injury, and cancer progression. The necrostatin family may serve as potential therapeutic targets for diseases such as ischemic/reperfusion of brain and myocardial infarction. Further insights into the signaling networks involved in the regulation of necroptosis will probably have important implications for the exploitation of necroptosis about the diagnosis or treatment of many diseases. Bile acids induced cell death also play important roles in the development of many diseases. Although there is no study indicating a direct relationship between bile acids and necroptosis, the assumption of this pathway should be considered carefully.

\section{Acknowledgement}

This work was supported by the Military Medical Youth Training Foundation (No. 13QNP119).

\section{References}

1. Savill J, Fadok V (2000) Corpse clearance defines the meaning of cell death Nature 407: 784-788.

2. Edinger AL, Thompson CB (2004) Death by design: apoptosis, necrosis and autophagy. Curr Opin Cell Biol 16: 663-669.

3. Taatjes DJ, Sobel BE, Budd RC (2008) Morphological and cytochemical determination of cell death by apoptosis. Histochem Cell Biol 129: 33-43.

4. Klionsky DJ, Emr SD (2000) Autophagy as a regulated pathway of cellular degradation. Science 290: 1717-1721.

5. Christofferson DE, Yuan J (2010) Necroptosis as an alternative form of programmed cell death. Curr Opin Cell Biol 22: 263-268.

6. Vandenabeele P, Declercq W, Vanden Berghe T (2008) Necrotic cell death and 'necrostatins': now we can control cellular explosion. Trends Biochem Sci 33 352-355.

7. Degterev A, Huang Z, Boyce M, Li Y, Jagtap P, et al. (2005) Chemical inhibitor of nonapoptotic cell death with therapeutic potential for ischemic brain injury Nat Chem Biol 1: 112-119.

8. Duprez L, Takahashi N, Van Hauwermeiren F, Vandendriessche B, Goossens V, et al. (2011) RIP kinase-dependent necrosis drives lethal systemic inflammatory response syndrome. Immunity 35: 908-918.

9. Peter ME (2011) Programmed cell death: Apoptosis meets necrosis. Nature 471: 310-312. 
Citation: Wanwei L, Menggang L, Long C, Fang W, Yao S, et al. (2014) Bile Acids Induced Cell Necropotosis. J Cytol Histol S4: 004. doi:10.4172/21577099.S4-004

10. Wang WJ, Wang Y, Chen HZ, Xing YZ, Li FW, et al. (2014) Orphan nuclear receptor TR3 acts in autophagic cell death via mitochondrial signaling pathway. Nat Chem Biol 10: 133-140.

11. Sosna J, Voigt S, Mathieu S, Kabelitz D, Trad A, et al. (2013) The proteases $\mathrm{HtrA} 2 / \mathrm{Omi}$ and UCH-L1 regulate TNF-induced necroptosis. Cell Commun Signal 11: 76.

12. Wu W1, Liu P, Li J (2012) Necroptosis: an emerging form of programmed cell death. Crit Rev Oncol Hematol 82: 249-258.

13. Li J, McQuade T, Siemer AB, Napetschnig J, Moriwaki K, et al. (2012) The RIP1/RIP3 necrosome forms a functional amyloid signaling complex required for programmed necrosis. Cell 150: 339-350.

14. Song BW, Wang L (2013) [Necroptosis: a programmed cell necrosis]. Sheng Li Ke Xue Jin Zhan 44: 281-286.

15. He S, Wang L, Miao L, Wang T, Du F, et al. (2009) Receptor interacting protein kinase-3 determines cellular necrotic response to TNF-alpha. Cell 137: 1100-1111.

16. Cho Y, Challa S, Chan FK (2011) A RNA interference screen identifies RIP3 as an essential inducer of TNF-induced programmed necrosis. Adv Exp Med Biol 691: 589-593.

17. Xie T, Peng W, Yan C, Wu J, Gong X, et al. (2013) Structural insights into RIP3mediated necroptotic signaling. Cell Rep 5: 70-78.

18. Khan N, Kate E Lawlor, James M Murphy, James E Vince, et al. (2014) More to life than death: molecular determinants of necroptotic and non-necroptotic RIPK3 kinase signaling. Current Opinion in Immunology 26: 76-89.

19. Chiang JY (2013) Bile acid metabolism and signaling. Compr Physiol 3: 1191 1212.

20. Rust C, Wild N, Bernt C, Vennegeerts T, Wimmer R, et al. (2009) Bile acidinduced apoptosis in hepatocytes is caspase-6-dependent. J Biol Chem 284 2908-2916.

21. Spivey JR, Bronk SF, Gores GJ (1993) Glycochenodeoxycholate-induced letha hepatocellular injury in rat hepatocytes. Role of ATP depletion and cytosolic free calcium. J Clin Invest 92: 17-24.

22. Hylemon PB, Zhou H, Pandak WM, Ren S, Gil G, et al. (2009) Bile acids as regulatory molecules. J Lipid Res 50: 1509-1520.

23. Higuchi H, Gores GJ (2003) Bile acid regulation of hepatic physiology: IV. Bile acids and death receptors. Am J Physiol Gastrointest Liver Physiol 284: G734-738.

24. Lee EW, Seo J, Jeong M, Lee S, Song J (2012) The roles of FADD in extrinsic apoptosis and necroptosis. BMB Rep 45: 496-508.

25. Matsubara T, Li F, Gonzalez FJ (2013) FXR signaling in the enterohepatic system. Mol Cell Endocrinol 368: 17-29.

26. Akare S, Martinez JD (2005) Bile acid induces hydrophobicity-dependent membrane alterations. Biochim Biophys Acta 1735: 59-67.

27. Barrasa JI, Olmo N, Lizarbe MA, Turnay J (2013) Bile acids in the colon, from healthy to cytotoxic molecules. Toxicol In Vitro 27: 964-977.
28. Gardès C, Chaput E, Staempfli A, Blum D, Richter H, et al. (2013) Differential regulation of bile acid and cholesterol metabolism by the farnesoid $X$ receptor in Ldlr -/- mice versus hamsters. J Lipid Res 54: 1283-1299.

29. Forman BM, Goode E, Chen J, Oro AE, Bradley DJ, et al. (1995) Identification of a nuclear receptor that is activated by farnesol metabolites. Cell 81: 687-693.

30. Bishop-Bailey D, Walsh DT, Warner TD (2004) Expression and activation of the farnesoid $X$ receptor in the vasculature. Proc Natl Acad Sci U S A 101 3668-3673.

31. Zhang Y, Kast-Woelbern HR, Edwards PA (2003) Natural structural variants of the nuclear receptor farnesoid $X$ receptor affect transcriptional activation. J Biol Chem 278: 104-110.

32. Huber RM, Murphy K, Miao B, Link JR, Cunningham MR, et al. (2002) Generation of multiple farnesoid-X-receptor isoforms through the use of alternative promoters. Gene 290: 35-43.

33. Joo JH, Jetten AM (2010) Molecular mechanisms involved in farnesol-induced apoptosis. Cancer Lett 287: 123-135.

34. Ohno T, Shirakami Y, Shimizu M, Kubota M, Sakai H, et al. (2012) Synergistic growth inhibition of human hepatocellular carcinoma cells by acyclic retinoid and GW4064, a farnesoid X receptor ligand. Cancer Lett 323: 215-222.

35. Feoktistova M, Geserick P, Kellert B, Dimitrova DP, Langlais C, et al. (2011) clAPs block Ripoptosome formation, a RIP1/caspase-8 containing intracellular cell death complex differentially regulated by cFLIP isoforms. Mol Cell 43: 449-463.

36. Cho YS, Challa S, Moquin D, Genga R, Ray TD, et al. (2009) Phosphorylationdriven assembly of the RIP1-RIP3 complex regulates programmed necrosis and virus-induced inflammation. Cell 137: 1112-1123.

37. Kang TB, Yang SH, Toth B, Kovalenko A, Wallach D (2013) Caspase-8 blocks kinase RIPK3-mediated activation of the NLRP3 inflammasome. Immunity 38 $27-40$

38. Welz PS, Wullaert A, Vlantis K, Kondylis V, Fernández-Majada V, et al. (2011) FADD prevents RIP3-mediated epithelial cell necrosis and chronic intestinal inflammation. Nature 477: 330-334.

39. Vandenabeele P, Galluzzi L, Vanden Berghe T, Kroemer G (2010) Molecular mechanisms of necroptosis: an ordered cellular explosion. Nat Rev Mol Cell Biol 11: 700-714.

40. Vanlangenakker N, Vanden Berghe T, Vandenabeele P (2012) Many stimuli pul the necrotic trigger, an overview. Cell Death Differ 19: 75-86.

41. Zhangxue H, Min G, Jinning Z, Yuan S, liW, etal. (2012) Glycochenodeoxycholate induces rat alveolar epithelial type II cell death and inhibits surfactant secretion in vitro. Free Radic Biol Med 53: 122-128.

42. Wu L, Li W, Wang Z, Yuan Z, Hyder Q (2013) Bile acid-induced expression of farnesoid $X$ receptor as the basis for superiority of internal biliary drainage in experimental biliary obstruction. Scand J Gastroenterol 48: 496-503.

This article was originally published in a special issue, Histology and Histopathology handled by Editor(s). Borislav A. Alexiev, University of Maryland Medical Center, USA 